\title{
Extended Signaling by Melatonin
}

\section{Hardeland $\mathbf{R}^{*}$}

Johann Friedrich Blumenbach Institute of Zoology and Anthropology, University of Goettingen, Germany

*Corresponding author: Rüdiger Hardeland, Johann Friedrich Blumenbach Institute of Zoology and Anthropology, University of Goettingen, Buergerstrasse 50, D-37073 Goettingen, Germany, Email: rhardel@gwdg.de

\section{Mini Review}

Volume 3 Issue 1

Received Date: April 24, 2018

Published Date: May 08, 2018

DOI: $10.23880 /$ cclsj-16000123

\section{Abstract}

The whole spectrum of melatonin's actions cannot be directly deduced from its primary signaling via its membranebound G protein-coupled receptors. This is not so much a matter of possibly existing other binding sites, but rather of the control of other important regulators by melatonin. Therefore, secondary signaling by factors influenced by melatonin has to be taken into consideration, such as other humoral factors, components of circadian oscillators, sirtuins, and microRNAs. Transmission of microRNAs by exosomes contributes to the actions of melatonin. In plants, melatoninbinding proteins are being investigated, but additionally interactions with phytohormones are likely. The recent demonstration of melatonin formation in mitochondria of both plants and mammals as well as the presence of a mammalian mitochondrial melatonin receptor open new aspects of melatonin's intracellular actions.

Keywords: Circadian; Melatonin; MicroRNAs; Mitochondria; Sirtuins

Abbreviations: GPCRs: G Protein-Coupled Receptors; RZR: Retinoid Z Receptor; CaM: Calmodulin; ASMT: Acetyl Serotonin Methyl Transferase; mtPTP: Mitochondrial Permeability Transition Pore; sAC: Soluble Adenylyl Cyclase; SCN: Suprachiasmatic Nucleus; AD: Alzheimer's Disease.

\section{Introduction}

In mammals, melatonin is known to mainly act via two membrane-bound, G protein-coupled receptors (GPCRs) [1-5]. According to actual terminology, these proteins are known as $\mathrm{MT}_{1}$ and $\mathrm{MT}_{2}$ receptors, whereas their genes have been named MTNR1A and MTNR1B, respectively. In nonmammalian vertebrates, a third membrane receptor, $\mathrm{Mel}_{1 \mathrm{c}}$, was discovered [6]. Apart from the GPCRs, several other melatonin binding sites were described, as summarized elsewhere [5]. One of these, originally believed to represent a third melatonin receptor found in the literature under the name of ' $\mathrm{MT}_{3}$ ', was later identified as the enzyme quinone reductase $2[7,8]$. The enzyme was also shown to bind other ligands with similar or even higher affinity, to be inhibited rather than activated by melatonin and, importantly, to be devoid of any demonstrable signaling pathway [9]. Therefore, it cannot be classified as a receptor. Countless publications have dealt with transcription factors of the retinoic acid receptor superfamily, subforms of ROR (retinoic acid receptor-related orphan receptor) and RZR (retinoid Z receptor), that were assumed to represent nuclear melatonin receptors. Although there was a lot of controversy about this issue from the beginning, the numerous studies in which such a role was taken as granted led to their consideration in many reviews until the last decade [5,9]. After the demonstration that the 


\section{Cell \& Cellular Life Sciences Journal}

most often discussed subform, ROR $\alpha$, does not bind melatonin, this debate seems to have ended $[10,11]$. ROR $\gamma$ was also shown to be devoid of melatonin binding, an evaluation of $\operatorname{ROR} \beta$ (= RZR $\beta$ ) is still missing, and other members of the superfamily have not been associated with melatonin [10]. Several other proteins, such as calreticulin and some poorly characterized nuclear proteins, one of them with homology to calreticulin, were also reported to bind melatonin, findings of potentially high interest [12]. However, the required detailed analysis of a melatonergic signal transduction pathway is still missing. As calreticulin displays various actions outside the endoplasmic reticulum and is, in some of its functions, related to the JAK/STAT pathway, a closer look by investigators may be worth-while. However, the connection between calreticulin and JAK/STAT has to date been limited to specific immunological effects, mainly in myelofibrosis, whereas no corresponding data on melatonin are available in a pertinent context $[13,14]$.

Another, in terms of regulation important protein, calmodulin ( $\mathrm{CaM})$, has also been shown to bind melatonin and to act as an inhibitor of CaM-dependent actions $[15,16]$. Although some affinity data seemed to suffice for physiological signaling, a discussion went on concerning the question of whether CaM contains a high- or a lowaffinity binding site. While the earlier studies indicated high affinity binding, later investigations concluded on much lower affinities in the micromolar range [9,15-18]. It should be briefly mentioned that melatonin was also reported to bind with low affinity to a membrane-bound variant of CaM in Xenopus oocytes [19]. Nevertheless, a physiological role of CaM as a melatonin-binding protein cannot be completely ruled out, especially as the affinity of melatonin to the CaM/CaM kinase II complex was considerably higher than that to CaM alone [20]. Therefore, melatonin binding to the complexes with CaM kinase II or other CaM-activated enzymes might be of much higher relevance.

\section{Melatonin Binding Sites Outside the Vertebrates}

As melatonin is a ubiquitous compound present in presumably all eukaryotes and many bacteria, its actions require binding sites in all these organisms [21-23]. In principle, actions via the also widely present CaM might be possible in all eukaryotes and this would be in accordance with the first effects described in plants and ciliates, concerning the cytoskeleton, in the years 19691973, although mechanistic details had not been elaborated at the time of discovery $[21,24]$. Beyond this possibility, there is an urgent demand for identifying high-affinity binding sites in non-vertebrates to understand melatonin's actions in these organisms. However, investigations of this type are still in their infancy. A high-affinity binding site with pharmacological similarity to the mammalian $\mathrm{MT}_{2}$ receptor was described in crayfish visual cells [25]. In Drosophila, a GPCR encoded by the $C G 4313$ gene was concluded to be responsible for effects of melatonin on cardiac rhythmicity [26]. However, one should be aware that melatonin levels are in this genus extremely low and only attain concentrations 3 orders of magnitude lower than those of its precursor, $\mathrm{N}$ acetylserotonin [27]. This difference is meanwhile explained by deficiency of the last enzyme in melatonin synthesis, $\mathrm{N}$-acetylserotonin $\mathrm{O}$-methyltransferase (ASMT). Therefore, the physiological meaning of a melatonin receptor in Drosophila remains questionable. Data obtained in Caenorhabditis elegans indicated the presence of a GPCR with pharmacological properties similar to $\mathrm{MT}_{1}$ [28]. In this study, effects by melatonin were shown to be interrupted by mutations in the genes of $\mathrm{G} \alpha$ subunits. In a dinoflagellate, Lingulodinium polyedrum, in which no classic melatonin receptors were detectable by standard procedures, the presence of binding sites was concluded on the basis of accumulation of external melatonin [29]. In plants, in which melatonin has become an important issue during the last years, the hunt for melatonin binding sites has meanwhile started [23,24,30,31]. Apart from a possible signaling via CaM, high-affinity binding sites have been described in studies on PR-10 proteins applying cocrystallization procedures. Proteins with these properties were discovered in St John's wort [32] \& Lupine [33]. A complication has resulted from the fact that these proteins also bind with substantial affinity cytokinins, a class of phytohormones. The relationship between melatonin and cytokinins awaits further elucidation. Anyway, the cross talks between melatonin and other phytohormones is, at least, complicated and not easily clarified. In several studies, melatonin seemed to exert auxin-like actions [34]. However, transcriptomic and proteomic data as well as other functional studies rather indicated relationships to different phytohormones, such as abscisic, jasmonic, and salicylic acids, including related volatile molecules, such as ethylene and methyl jasmonate $[24,30,31,35]$. The upregulation of genes associated with actions of abscisic, jasmonic and salicylic acids may be taken as a hint for secondary melatonin signaling via these phytohormones, especially in the context of stress responses. Clarification of the relationships promises substantial new insights on melatonin's actions and signaling pathways in plants. 


\section{Cell \& Cellular Life Sciences Journal}

\section{A Melatonin Receptor and Other Binding Sites in Mitochondria}

For multiple reasons, mitochondria have been in the focus of melatonin research. These include the role of these organelles as sources of free radicals, their sensitivity to oxidative damage, their implication in apoptosis, the consequences of mitophagy and peripheral mitochondrial depletion, their participation in metabolic sensing, the existence of numerous mitochondrial diseases, their role in aging, and various mitochondria protecting actions of melatonin [9,36-48]. More recently, several important and, for some time, unforeseen developments have taken place in this field. One of the puzzling aspects of melatonin in mitochondria concerned the identification of a binding site with lower affinity compared to the GPCRs [49]. In that study, melatonin was shown to directly inhibit the opening of the mitochondrial permeability transition pore (mtPTP). Meanwhile, the role of melatonin does not seem to serve the complete inhibition of superoxide flashes released through this pore, but rather to reduce their duration [50]. However, a $\mathrm{K}_{\mathrm{i}}$ value of $0.8 \mu \mathrm{M}$ indicated a requirement for melatonin uptake into these organelles if this effect should be of physiological relevance. In fact, such an uptake was demonstrated in other investigations [51,52]. More recently, accumulation of melatonin became more probable by the involvement of transporters in melatonin uptake, in particular, the oligopeptide transporters PEPT1/2 [53]. However, this was not yet the end of the story. Starting from the insight that melatonin has been originated during evolution in bacteria, including $\alpha$ proteobacteria, the group that was ancestral to mitochondria [54], melatonin synthesis was, in fact, demonstrated in plant mitochondria [55]. Finally, melatonin synthesis was recently demonstrated in the mitochondrial matrix of the murine brain [56]. Contrary to pineal melatonin synthesis, no substantial circadian differences were observed in the brain mitochondria, a finding that is not really surprising because a similar paucity of circadian fluctuations was also observed in other extrapineal sites of melatonin formation [57]. Notably, melatonin is formed in numerous tissues and cells [9,57-59]. Therefore, mitochondrial production in conjunction with uptake from the circulation might well generate mitochondrial concentrations that suffice for physiological actions in these organelles. Despite the fact that the earlier discussed but not approved nuclear receptors have failed to explain intracellular actions of melatonin, its mitochondrial synthesis underlines the likelihood of until recently unknown intracellular properties and signaling. This assumption is supported by the presence of the melatonin receptor $\mathrm{MT}_{1}$ in mitochondria. The original demonstration has been confirmed in the article on mitochondrial melatonin synthesis $[56,60]$.The fact that mitochondria are the sites of both synthesis and action has led to the proposal of the new term "automitocrine" [56]. In this latter study, $\mathrm{MT}_{1}$ was reported to be localized in the outer mitochondrial membrane, to interact with $G$ proteins and to associate with $\beta$-arrestins. Its function was seen in the inhibition of cytochrome $c$ release and caspase activation, effects known since long from exogenously delivered melatonin, but which had previously not been understood in mechanistic terms. Overexpression of the mitochondrial $\mathrm{MT}_{1}$ receptor was also reported to exert neuroprotective effects [56].

Actually, the question remains whether an $\mathrm{MT}_{1}$ receptor in the outer mitochondrial membrane will explain all other melatonin effects in these organelles, especially those concerning the regulation of electron flux and of processes in the matrix. Concerning the regulation of electron entrance at Complex 1, a melatonin binding site had been assumed to exist at the amphipathic ramp of this complex, which extends into the matrix. High affinity binding had been provisionally communicated, but detailed experimental data had never been published. These findings, cited elsewhere, cannot yet be judged with certainty [38]. Moreover, the presence of $\mathrm{MT}_{1}$ in the outer mitochondrial membrane cannot easily explain other melatonin-associated effects on respiratory electron flux, especially if these do not only correct oxidotoxic actions by mitotoxins. Therefore, the additional presence of $\mathrm{MT}_{1}$ in the inner membrane, perhaps in other cell types, might be taken into consideration. This had been suggested in analogy to findings on respiration effects of cannabinoids via the mitochondrial cannabinoid receptor mtCB1 that acts via a $G_{i}$ protein at the matrix side of the inner membrane and signal transduction by inhibiting the soluble adenylyl cyclase (sAC) and PKA in the matrix [61]. Whether or not this possible pathway of melatonin signaling exists requires experimental support [62].

\section{Secondary Signaling via Sirtuins}

Being a highly pleiotropic regulator molecule [9], melatonin also influences, directly or indirectly, positively or negatively, various other important control factors. Among them are other hormones, the excitatory and proinflammatory mediator nitric oxide [42], cytokines [9,63-65], and various metabolic sensors [42]. A specific group of molecules, the sirtuins, have recently attracted particular interest in melatonin research. Having been 


\section{Cell \& Cellular Life Sciences Journal}

detected in the context of aging, sirtuins turned out to possess a remarkably broad spectrum of actions that reaches from metabolic sensing to mitochondrial effects, anti-inflammatory properties and, importantly, participation in the functioning of cellular circadian oscillators $[42,64,66,67]$. For two reasons, this latter aspect is of particular significance for the understanding of melatonin's actions. First, melatonin's functions are intertwined with circadian rhythms because the pineal hormone is under circadian control but, in reverse, influences central and peripheral oscillators $[66,68]$. Second, circadian oscillators control countless functions in almost all nucleate cells. Therefore, influences of melatonin on sirtuins have to be transduced to countless functions much beyond traditional awareness.

At the beginning of sirtuin studies in melatonin research, a controversy seemed to emerge, which was, however, not that much controversial as soon as the dynamic properties of circadian oscillators were taken into account. Studies in cancer had revealed that melatonin strongly downregulated sirtuin 1 (SIRT1) levels $[69,70]$. However, just the opposite, namely, upregulation of SIRT1 by melatonin, was observed in nontumor cells, as recently summarized [66]. These stimulatory effects were obtained in the context of aging and also under conditions of inflammation. For several reasons, these findings are of particular interest. First, sirtuins had been discovered as aging suppressors and, although some divergent effects of SIRT1 on aging had been reported, life extension by brain-specific SIRT1 overexpression has been documented [71]. Second, the parallel declines of melatonin levels and SIRT1 expression, as observed during aging, may be taken as a hint for a functional relationship [67]. Third, the fields of action of melatonin and SIRT1 are considerably overlapping, in terms of anti-inflammatory effects, mitochondrial protection, enhancement of circadian amplitudes [64,66,67,72], and anti-aging effects $[42,71,73]$. Notably, the lifespan extending effect of melatonin was more pronounced in the senescenceaccelerated mouse strain SAMP8 than in the normally aging control strain SAMR1, a finding that should be seen in relation to the typical underexpression of SIRT1 in untreated SAMP8 mice $[73,74]$.

The divergent results obtained in tumor and nontumor cells turned out to not be really contradictory, because of the decisive role of SIRT1 in acting as an accessory component of circadian oscillators with amplitudeenhancing properties and a fundamental difference concerning the expression of some core oscillator genes with antitumor potential $[65,66,75]$. As outlined in these publications, tumor suppressor genes have to be epigenetically silenced to allow the existence as a tumor cell. This is especially required for the gene Per2 (Period 2), whose deficiency makes a mouse cancer-prone, and which is suppressed in the cancer lines studied in the context of melatonin and SIRT1 [76]. As a result, the oscillators of cancer cells are strongly dysregulated and more or less fixed in abnormal phase positions in which proliferation is favored. Under these conditions, under which Per2 is silenced, the core oscillator protein CLOCK and also SIRT1 are strongly upregulated, a change that is gradually reversed by melatonin $[65,66,75]$.

The relationship between melatonin and SIRT1 will certainly require further elucidation. However, recent data indicate that SIRT1 may be a downstream factor of melatonin, not only because of the multiply documented upregulation of SIRT1 in nontumor cells, but even more as some effects of melatonin were shown to be blunted by SIRT1 inhibition [66]. This was shown in a study on counteractions of glucocorticoid-induced chondrocyte impairment by melatonin [77]. Similar conclusions were drawn in different models of neuroprotection [78-80]. As a full record of findings would exceed the scope of this article, only a few important findings shall be addressed. Protective effects by melatonin were counteracted by the sirtuin inhibitors sirtinol [81] and EX527 [78]. Melatonin did not only enhance, in the septic heart, SIRT1 expression, but also $\mathrm{NAD}^{+}$levels, which determine SIRT1 activity, and expression of Nampt mRNA, which encodes the rate-limiting enzyme of $\mathrm{NAD}^{+}$formation, nicotinamide phosphoribosyltransferase [82]. Moreover, this study underlined the common anti-inflammatory properties of melatonin and SIRT1 in the context of sepsis. Finally, this investigation also revealed upregulation of Rora and downregulation of Rev-erb $\alpha$ mRNAs, i.e., an activator and a repressor at the ROR response element (RORE), respectively [82]. These findings indicate enhanced expression of genes with RORE-containing promoters, such as Bmal1 and Clock, i.e., core oscillator genes whose proteins stimulate the expression of, e.g., Per2, other Per and Cry (cryptochrome) genes and Nampt as well. Beyond the pathologies, it should be noted that melatonin also upregulated Sirt1 mRNA in PMBCs of healthy control subjects [83].

Collectively, findings indicate that, at least, some of melatonin's actions are mediated by SIRT1. This signifies the extension of receptor-mediated signaling by secondary processes that depend on this sirtuin and have to be taken into consideration in future studies. These 


\section{Cell \& Cellular Life Sciences Journal}

effects on SIRT1 also imply secondary actions via the circadian oscillator system. Importantly, these secondary circadian changes are not restricted to the circadian master clock, the suprachiasmatic nucleus (SCN), but extend too many, perhaps, all peripheral oscillators. This would be in accordance with some earlier observations and expand the spectrum of chronobiological actions of melatonin considerably [68]. It seems to be of fundamental relevance to be aware of the countless functions driven by circadian oscillators via rhythmic expression of circadian controlled genes [84].

Whether or not SIRT1 is the only sirtuin that transmits information originating from melatonin remains an issue of actuality, but will require further experimental support. This may concern especially the mitochondrially located SIRT3 and the constitutively chromatin-associated SIRT6. Both SIRT3 and SIRT6 are driven by the $\mathrm{NAD}^{+}$cycle and transmit circadian information $[85,86]$, but, because of their localization, they may not feed back to core oscillators. Meanwhile, upregulation of SIRT3 expression by melatonin has been reported for ovary/oocytes [8789], liver [90], and heart [91-93], under various pathological conditions. The actions on SIRT3 were associated with signaling via PGC- $1 \alpha[90,91]$, a property shared with SIRT1 [94].

\section{Secondary Signaling via MicroRNAs and Exosomes}

MicroRNAs (miRNAs) have received increasing attention during the last years, for several reasons [9598]. First, they act as co-regulators of countless functions, mainly by causing destruction of target mRNAs via formation of a microRNA-induced silencing complex (RISC complex) that leads to the destruction of the double-stranded intermediate. Their formation can take place in different ways, either from longer hairpin transcripts known as pri-miRNAs that are converted to pre-miRNAs, which are spliced in the cytoplasm by the RNase III Dicer and, thereafter, unwound. Alternately, other miRNAs are formed from intron sequences by hnRNA splicing via intermediates known as mirtrons [99]. Moreover, some miRNAs are subject to RNA editing $[100,101]$. In addition to mRNA targeting, some miRNAs bind to a toll-like receptor, TLR7, which represents a protein that interacts exclusively with single-stranded RNAs, whereas other TLR subforms that are specialized for double-stranded RNAs do not interact with miRNAs [102-104]. A second reason concerning their importance is their abundance. In mammals, the number of miRNAs is estimated to be in the range of a thousand. As many of them are capable of targeting sometimes more than a hundred mRNAs, their impact may concern more than half of the genome [95-99]. A third reason is related to their distribution. In addition to actions in the cells in which they have been formed and to the possibility of entering neighbor cells via gap junctions, they are also distributed within the body by means of exosomes and ectosomes. Formally, exosomes and ectosomes are distinguished by their mode of biogenesis, either, in the former case, involving the multivesicular body and release by exocytosis or, in the latter case, by budding at the plasma membrane $[105,106]$. Despite this difference, ectosomes, also often referred to as microvesicles, and exosomes are frequently not clearly distinguished in experimental studies. However, regardless of the missing distinction, both of them are carriers of miRNAs, which are enriched in these extracellular vesicles by virtue of lncRNAs (long noncoding RNAs) and circRNAs (circular RNAs), which act as miRNA sponges [106-108]. As exosomes and ectosomes are found in all body fluids, they transmit regulatory messages over long distances to target cells. By virtue of this property, they are discussed in terms of spreading dysregulatory information of pathologies, but may also spread protective, correcting information upon curative treatment [109].

With regard to the pleiotropy of melatonin [9], numerous actions of this regulator molecule should be expected to be either transmitted or modulated by miRNAs. Actually available information clearly supports this conclusion. However, melatonin's spectrum of action is that large that a coherent picture concerning the participation of miRNAs cannot be easily achieved. Nevertheless, results obtained to date have brought about a remarkable number of important details. Instead of summarizing many, often functionally unrelated findings, the focus shall be laid on results of more fundamental relevance, which may also serve as a proof of principle for the involvement of miRNAs in melatonin signaling. Various other details have been reviewed elsewhere $[72,109]$. With regard to well-documented protective actions by melatonin, studies have often focused on the correction of detrimental effects. In a model of neuroinflammation by LPS (bacterial lipopolysaccharide), upregulation of miR-34a and downregulation of miR-146a and miR-126 have been reversed by melatonin, notably in conjunction with SIRT1 upregulation [80]. This effect may be indicative of the transmission of a melatonin signal via SIRT1 at the miRNA level. The observation of both up- and downregulations of different miRNAs was also made in models of Alzheimer's disease (AD), in which the pathological change consisted in upregulation of miR-124 


\section{Cell \& Cellular Life Sciences Journal}

and downregulation of miR-132 [110,111]. The correction of these effects was especially of importance in the case of the $A \beta$-induced suppression of miR-132, since this microRNA displays anti-apoptotic properties [111]. In a study on 25 AD-specific changes of miRNA expression, associations with melatonin were detected, among them a particularly strong connection with miR-765 and a negative one with miR-30e-5p [112]. It will be of great interest to have to whole set of these associations tested in a functional context, in animal models or clinically.

A few papers have addressed the effects of melatonin on stem cells, however, in poorly comparable models, such as hair follicle cells [113], cardiac progenitor cells [114] and mesenchymal stem cells [115]. Additional findings in spermatogonial cell lines are discussed elsewhere [109]. Collectively, more than 180 miRNAs were shown to be differentially expressed under the influence of melatonin. Two particular findings shall be specifically addressed. In cardiac progenitor cells exposed to hydrogen peroxide, melatonin maintained the expression of miR-675 by protecting its IncRNA precursor, H19 [114]. In mesenchymal stem cells, in which melatonin is known to induce chondrogenic and, under respective conditions, osteogenic differentiation [42], the pineal hormone was recently shown to induce two pro-chondrogenic miRNAs, miR-525b-3p and miR590-5p [115].

Another line of experimentation concerned the effect of melatonin on miRNAs in cancer cells, in particular, prostate and breast cancer cells, in which on costatic actions of melatonin had been repeatedly shown, and glioma cell lines [116-118]. In functional terms, melatonin inhibited angiogenesis via miR-3195 and miR-374b expression in prostate cancer, which should be of relevance to tumor growth [116]. In glioma cell lines, melatonin downregulated miR-155, which is believed to reduce proliferation and migration [118]. The broad scale study in breast cancer cells revealed differential expression in 22 miRNAs, which, according to $5^{\prime}$-utr sequencing, may target over 2000 mRNAs [117].

Although miRNAs are present in exosomes and ectosomes, changes in their RNA contents have not yet been studied in the context of melatonin. However, an investigation on pathological changes in exosomes and their counteraction by melatonin indicates a promising new line of experimentation. In vitro differentiated macrophages were exposed to exosomes from hepatocellular carcinoma cells, which had either remained untreated or were incubated with melatonin
[119]. In the recipient macrophages, exosomes from untreated carcinoma cells upregulated the secretion of various cytokines, such as IL-6, IL-10, IL-1 $1 \beta$, and TNF- $\alpha$, whereas these were downregulated by exosomes from melatonin-treated cells. These results demonstrate the potential of such an approach, but, in the future, the conclusiveness would be substantially enhanced by analyses of miRNA contents as well as of their sponges, lncRNAs and circRNAs.

\section{Conclusion}

Primary signaling via GPCRs [1-4] is beyond doubt in vertebrates. This statement also includes modulations via the nonbinding melatonin receptor ortholog GPR50, the stabilization of $\mathrm{MT}_{1}$ by the scaffolding protein MUPP1 (multi-PDZ domain protein 1), effects of homo- and heterodimerizations, and all consequences of receptor modification [5]. Whether or not CaM contributes in a substantial way to melatonin signaling in vertebrates may require further support, but this should remain to be considered in plants. Other binding sites in vertebrates have either not been approved, not been sufficiently studied in detail, or are devoid of a demonstrable signal transduction pathway.

However, the message of this mini reviews it to direct readers' attention to the manifold possibilities of secondary signaling. Regarding a highly pleiotropic molecule like melatonin, it is not surprising that it also acts on other powerful regulators, including other hormones, metabolic sensors, cellular co-regulators, and components of the circadian oscillators. As examples of secondary signaling by humoral factors, cytokines released by immune cells as well as uroguanylin and guanylin in the intestine should be mentioned $[63,65]$. These latter compounds are released by Paneth cells in response to $\mathrm{MT}_{2}$ activation and stimulate cGMP formation via the receptor guanylyl cyclase $2 \mathrm{C}$ (GUCY2C) in the plasma membrane of enterocytes [120-122]. A full spectrum of hormones and hormone-like compounds influenced by melatonin would exceed the scope of this article and would be worth another review.

In plants, high-affinity binding sites and primary signaling are awaiting elucidation, apart from the possibility of additional CaM-mediated effects. Despite this still existing gap, the interference of melatonin with genes under control of established phytohormones may indicate secondary signaling via these agents $[24,30,34,35]$. How melatonin may interact with these phytohormones remains to be clarified. 


\section{Cell \& Cellular Life Sciences Journal}

In mammals, signaling via mitochondrial $\mathrm{MT}_{1}$ opens an important new field of investigation, along with the fact of mitochondrial melatonin synthesis [56]. As melatonin is also formed in plant mitochondria [55], the possibility of intramitochondrial signaling in plants may be of interest, too, but would first require the identification of binding sites. In plants, melatonin formed in chloroplasts and its signaling deserve additional attention.

Secondary signaling via SIRT1 appears to be of fundamental importance. This is not only related to the more or less concomitant declines of melatonin and SIRT1 in the course of aging, but has also to be seen in the context of metabolic sensing and the circadian role of SIRT1. It will be of utmost importance to identify in the next future the extent of melatonin effects transmitted by SIRT1. Moreover, investigators who administer melatonin in experiments designed for cell protection should be particularly aware of the possibility of secondary signaling by SIRT1. This should include the identification of downstream factors controlled by SIRT1 as well as substrate proteins that are deacetylated by this enzyme. The upregulation of SIRT1 by melatonin, as already demonstrated in a number of cases, has to be taken into account in all aspects related to circadian rhythmicity [66,77-83]. The circadian dynamics is of fundamental significance for numerous physiological and cell biological parameters, already with regard to the countless circadian controlled genes and the global epigenetic regulation by circadian oscillators [84].

Attention concerning secondary signaling by SIRT1 should also include the possibility of changes in SIRT1controlled miRNAs. With regard to noncoding RNAs, the situation may be extremely complex, as other miRNAs and sponging RNAs may be also directly influenced by melatonin or by other components of circadian oscillators. A poorly investigated but highly promising field is that of signaling via exosomes and ectosomes. Whether directly or indirectly modulated by melatonin, the possible spreading of protective, perhaps, curative messages by these extracellular vesicles opens fascinating perspectives.

To date, one of the practical problems of noncoding RNA research consists in the countless number of different miRNAs, the various IncRNAs and circRNAs, and the even higher numbers of potentially targeted mRNAs [123]. Some investigators have concentrated their studies on a very few miRNAs, whereas others have chosen global RNA sequencing approaches. In both cases, the information remains limited, either because of the small molecular spectrum looked at or because of the difficulty of finding the decisive needle in the haystack. A way out may be to consequently concentrate first on approaches concerning effects of melatonin in a nonpathological context or in selecting treatments that are devoid of a foreseeably high complexity. For instance, the use of LPS or of mitochondrial toxins that exert countless effects in the entire body will presumably reveal a high number of changes, but a low degree of insight. This statement should not be misunderstood in terms of underrating the importance of pathophysiological studies, but rather indicate routes towards a better understanding of effects by melatonin per se, as far as secondary signaling is concerned.

\section{References}

1. Reppert SM, Weaver DR, Ebisawa T (1994) Cloning and characterization of a mammalian melatonin receptor that mediates reproductive and circadian responses. Neuron 13(5): 1177-1185.

2. Reppert SM, Godson, Mahle CD, Weaver DR, Slaugenhaupt SA, et al. (1995) Molecular characterization of a second melatonin receptor expressed in human retina and brain: the $\operatorname{Mel}_{1 b}$ melatonin receptor. Proc Natl Acad Sci USA 92(19): 8734-8738.

3. Jin X, von Gall C, Pieschl L, Gribkoff VK, Stehle JH, et al. (2003) Targeted disruption of the mouse Mel $_{1 \mathrm{~b}}$ melatonin receptor. Mol Cell Biol 23(3): 1054-1060.

4. Dubocovich ML, Markowska M (2005) Functional $\mathrm{MT}_{1}$ and $\mathrm{MT}_{2}$ melatonin receptors in mammals. Endocrine 27(2): 101-110.

5. Hardeland R (2009) Melatonin: Signaling mechanisms of a pleiotropic agent. BioFactors 35(2): 183-192.

6. Jockers R, Petit L, Lacroix I, de Coppet P, Barrett P, et al. (1997) Novel isoforms of Mel $_{1 c}$ melatonin receptors modulating intracellular cyclic guanosine 3',5'-monophosphate levels. Mol Endocrinol 11(8): 1070-1081.

7. Nosjean O, Ferro M, Coge F, Beauverger P, Henlin J-M, et al. (2000) Identification of the melatonin-binding site MT3 as the quinone reductase 2. J Biol Chem 275(40): 31311-31317. 


\section{Cell \& Cellular Life Sciences Journal}

8. Mailliet F, Ferry G, Vella F, Berger S, Coge F, et al. (2005) Characterization of the melatoninergic MT3 binding site on the NRH:quinone oxidoreductase 2 enzyme. Biochem Pharmacol 71(1-2): 74-78.

9. Hardeland R, Cardinali DP, Srinivasan V, Spence DW, Brown GM, et al. (2011) Melatonin - A pleiotropic, orchestrating regulator molecule. Prog Neurobiol 93(3): 350-384.

10. Slominski AT, Kim TK, Takeda Y, Janjetovic Z, Brozyna $\mathrm{AA}$, et al. (2014) ROR $\alpha$ and ROR $\gamma$ are expressed in human skin and serve as receptors for endogenously produced noncalcemic 20-hydroxy- and 20,23dihydroxyvitamin D. FASEB J 28: 2775-2789.

11. Slominski AT, Zmijewski MA, Jetten AM (2016) ROR alpha is not a receptor for melatonin. Bioessays 38(12):1193-1194.

12. Macias M, Escames G, Leon J, Coto A, Sbihi Y, et al. (2003) Calreticulin - melatonin. An unexpected relationship. Eur J Biochem 270: 832-840.

13. Guglielmelli P, Bartalucci N, Rotunno G, Vannucchi AM (2014) Calreticulin: a new horizon for the testing and treatment of myeloproliferative neoplasms. Expert Rev Hematol 7(4): 423-425.

14. Alshemmari SH, Rajan R, Emadi A (2016) Molecular pathogenesis and clinical significance of driver mutations in primary myelofibrosis: A review. Med Princ Pract 25(6): 501-509.

15. Benitez-King G, Huerto-Delgadillo L, Anton-Tay F (1993) Binding of ${ }^{3} \mathrm{H}$-melatonin to calmodulin. Life Sci 53(3): 201-207.

16. Benitez-King G, Anton-Tay F (1993) Calmodulin mediates melatonin cytoskeletal effects. Experientia 49(8): 635-641.

17. Turjanski AG, Estrin DA, Rosenstein RE, McCormick JE, Martin SR, et al. (2004) NMR and molecular dynamics studies of the interaction of melatonin with calmodulin. Protein Sci 13(11): 2925-2938.

18. Turjanski AJ, Estrin DA, Rosenstein RE, Mccormick JE, Martin SR, et al. (2007) The interaction between melatonin and calmodulin: a NMR and molecular dynamics study. In: Melatonin - From Molecules to Therapy (Pandi-Perumal SR, Cardinali DP, Eds); Nova Science, New York, pp: 47-68.
19. Paz Romero M, García-Pergañeda A, Guerrero JM, Osuna C (1998) Membrane-bound calmodulin in Xenopus laevis oocytes as a novel binding site for melatonin. FASEB J 12(13): 1401-1408.

20. Landau M, Zisapel N (2007) The low affinity binding of melatonin to calmodulin: use of computational methods to explain its physiological relevance. In: Melatonin - From Molecules to Therapy (PandiPerumal SR, Cardinali DP, Eds); Nova Science, New York, pp: 69-79.

21. Hardeland R, Fuhrberg B (1996) Ubiquitous melatonin - Presence and effects in unicells, plants and animals. Trends Comp Biochem Physiol 2: 25-45.

22. Hardeland R, Poeggeler B (2003) Non-vertebrate melatonin. J Pineal Res 34(4): 233-241.

23. Tan DX, Hardeland R, Manchester LC, Korkmaz A, Ma $\mathrm{S}$, et al. (2012) Functional roles of melatonin in plants and perspectives in nutritional and agricultural science. J Exp Bot 63(2): 577-597.

24. Hardeland R (2016) Melatonin in plants - diversity of levels and multiplicity of functions. Front Plant Sci 7: 198.

25. Mendoza-Vargas L, Solís-Chagoyán $H$, Benítez-King G, Fuentes-Pardo B (2009) $\mathrm{MT}_{2}$-like melatonin receptor modulates amplitude receptor potential in visual cells of crayfish during a 24-hour cycle. Comp Biochem Physiol A Mol Integr Physiol 154(4): 486-492.

26. VanKirk T, Powers E, Dowse HB (2017) Melatonin increases the regularity of cardiac rhythmicity in the Drosophila heart in both wild-type and strains bearing pathogenic mutations. J Comp Physiol B 187(1): 63-78.

27. Callebert J, Jaunay JM, Jallon JM (1991) Control of Drosophila biorhythms. Adv Pineal Res 5: 81-84.

28. Tanaka D, Furusawa K, Kameyama K, Okamoto H, Doi M (2007) Melatonin signaling regulates locomotion behavior and homeostatic states through distinct receptor pathways in Caenorhabditis elegans. Neuropharmacology 53(1): 157-168.

29. Hardeland R, Pandi-Perumal SR, Poeggeler B (2007) Melatonin in plants - Focus on a vertebrate night hormone with cytoprotective properties. Funct Plant Sci Biotechnol 1(1): 32-45. 


\section{Cell \& Cellular Life Sciences Journal}

30. Hardeland R (2015) Melatonin in plants and other phototrophs - advances and gaps concerning the diversity of functions. J Exp Bot 66(3): 627-646.

31. Hardeland R (2016) Melatonin - another phytohormone? J Bot Sci 5(2): 20-23.

32. Sliwiak J, Dauter Z, Jaskolski M (2016) Crystal structure of Hyp-1, a Hypericum perforatum PR-10 protein, in complex with melatonin. Front Plant Sci 7:668.

33. Sliwiak J, Sikorski M, Jaskolski M (2018) PR-10 proteins as potential mediators of melatonincytokinin cross-talk in plants: crystallographic studies of LIPR-10.2B isoform from yellow lupine. FEBS J [Epub ahead of print, Apr 6]; doi: 10.1111/febs.14455.

34. Arnao MB, Hernández-Ruiz J (2015) Functions of melatonin plants: a review. J Pineal Res 59(2): 133150.

35. Weeda S, Zhang N, Zhao X, Ndip G, Guo Y, et al. (2014) Arabidopsis transcriptome analysis reveals key roles of melatonin in plant defense systems. PLoS One 9(3): e93462.

36. Acuña-Castroviejo D, Escames G, León J, Carazo A, Khaldy H (2003) Mitochondrial regulation by melatonin and its metabolites. Adv Exp Med Biol 527: 549-557.

37. Acuña-Castroviejo D, Escames G, Rodríguez MI, López LC (2007) Melatonin role in the mitochondrial function. Front Biosci 12: 947-963.

38. Hardeland R (2009) Melatonin, mitochondrial electron flux and leakage: recent findings and resolution of contradictory results. Adv Stud Biol 1(5): 207-230.

39. Hardeland R, Poeggeler B, Pappolla MA (2009) Mitochondrial actions of melatonin - an endeavor to identify their adaptive and cytoprotective mechanisms. Proc Saxon Acad Sci 65(3): 14-31.

40. Carretero M, Escames G, López LC, Venegas C, Dayoub JC, et al. (2009) Long-term melatonin administration protects brain mitochondria from aging. J Pineal Res 47(2): 192-200.
41. Hardeland R (2009) Neuroprotection by radical avoidance: search for suitable agents. Molecules 14(12): 5054-5102.

42. Hardeland R (2013) Melatonin and the theories of aging: a critical appraisal of melatonin's role in antiaging mechanisms. J Pineal Res 55(4): 325-356.

43. Paradies G, Paradies V, Ruggiero FM, Petrosillo G (2017) Mitochondrial bioenergetics decay in aging: beneficial effect of melatonin. Cell Mol Life Sci 74(21): 3897-3911.

44. Wongprayoon P, Govitrapong P (2017) Melatonin as a mitochondrial protector in neurodegenerative diseases. Cell Mol Life Sci 74(21): 3999-4014.

45. Cardinali DP, Vigo DE (2017) Melatonin, mitochondria, and the metabolic syndrome. Cell Mol Life Sci 74(21): 3941-3954.

46. Reiter RJ, Rosales-Corral S, Tan D-X, Jou MJ, Galano A, et al. (2017) Melatonin as a mitochondria-targeted antioxidant: one of evolution's best ideas. Cell Mol Life Sci 74(21): 3863-3881.

47. Ahluwalia A, Brzozowska IM, Hoa N, Jones MK, Tarnawski AS (2018) Melatonin signaling in mitochondria extends beyond neurons and neuroprotection: Implications for angiogenesis and cardio/gastroprotection. Proc Natl Acad Sci USA 115(9): E1942-E1943.

48. Reiter RJ, Tan D-X, Rosales-Corral S, Galano A, Zhou $\mathrm{XJ}$, et al. (2018) Mitochondria: Central organelles for melatonin's antioxidant and anti-aging actions. Molecules 23 (2): E509.

49. Andrabi SA, Sayeed I, Siemen D, Wolf G, Horn TF (2004) Direct inhibition of the mitochondrial permeability transition pore: a possible mechanism responsible for anti-apoptotic effects of melatonin. FASEB J 18(7): 869-871.

50. Jou MJ (2011) Melatonin preserves the transient mitochondrial permeability transition for protection during mitochondrial $\mathrm{Ca}^{2+}$ stress in astrocyte. J Pineal Res 50(4): 427-435.

51. Messner M, Hardeland R, Rodenbeck A, Huether G (1998) Tissue retention and subcellular distribution of continuously infused melatonin in rats under near physiological conditions. J Pineal Res 25(4): 251-259. 


\section{Cell \& Cellular Life Sciences Journal}

52. López A, García JA, Escames G, Venegas C, Ortíz F, et al. (2009) Melatonin protects the mitochondria from oxidative damage reducing oxygen consumption, membrane potential, and superoxide anion production. J Pineal Res 46(2): 188-198.

53. Mayo JC, Sainz RM, González-Menéndez P, Hevia D, Cernuda-Cernuda R (2017) Melatonin transport into mitochondria. Cell Mol Life Sci 74(21): 3927-3940.

54. Tan D-X, Manchester LC, Liu X, Rosales-Corral SA, Acuna-Castroviejo D, et al. (2013) Mitochondria and chloroplasts as the original sites of melatonin synthesis: a hypothesis related to melatonin's primary function and evolution in eukaryotes. J Pineal Res 54(2): 127-138.

55. Wang L, Feng C, Zheng X, Guo Y, Zhou F, et al. (2017) Plant mitochondria synthesize melatonin and enhance the tolerance of plants to drought stress. J Pineal Res 63(3): e12429.

56. Suofu Y, Li W, Jean-Alphonse FG, Jia J, Khattar NK, et al. (2017) Dual role of mitochondria in producing melatonin and driving GPCR signaling to block cytochrome c release. Proc Natl Acad Sci USA 114(38): E7997-E8006.

57. Hardeland R (2017) The expanding functions of melatonin and their consequences to signaling. In: Mini-Reviews in Recent Melatonin Research (Hardeland R, Ed), Cuvillier, Göttingen, Germany, pp: 26-42.

58. Acuña-Castroviejo D, Escames G, Venegas C, DíazCasado ME, Lima-Cabello E, et al. (2014) Extrapineal melatonin: sources, regulation, and potential functions. Cell Mol Life Sci 71(16): 2997-3025.

59. Hardeland R (2017) Melatonin - More than just a pineal hormone. Biomed J Sci Tech Res 1(4): 00351.

60. Wang X, Sirianni A, Pei Z, Cormier K, Smith K, et al. (2011) The melatonin MT1 receptor axis modulates mutant Huntingtin-mediated toxicity. J Neurosci 31(41): 14496-14507.

61. Hebert-Chatelain E, Desprez T, Serrat R, Bellocchio L, Soria-Gomez E, et al. (2016) A cannabinoid link between mitochondria and memory. Nature 539(7630): 555-559.

62. Hardeland R (2017) Melatonin and the electron transport chain. Cell Mol Life Sci 74(21): 3883-3896.
63. Carrillo-Vico A, Lardone PJ, Álvarez-Sánchez N, Rodríguez-Rodríguez A, Guerrero JM (2013) Melatonin: buffering the immune system. Int J Mol Sci 14(4): 8638-8683.

64. Hardeland R, Cardinali DP, Brown GM, Pandi-Perumal SR (2015) Melatonin and brain inflammaging. Prog Neurobiol 127-128: 46-63.

65. Hardeland R (2016) Opposite effects of melatonin in different systems and under different conditions. Curr Top Biochem Res 17: 57-69.

66. Hardeland R (2017) Melatonin and the pathologies of weakened or dysregulated circadian oscillators. J Pineal Res 62(1): e12377.

67. Hardeland R (2018) Brain inflammaging: roles of melatonin, circadian clocks and sirtuins. J Clin Cell Immunol 9(1): 543.

68. Hardeland R, Madrid JA, Tan DX, Reiter RJ (2012) Melatonin, the circadian multioscillator system and health: the need for detailed analyses of peripheral melatonin signaling. J Pineal Res 52(2): 139-166.

69. Jung-Hynes B, Nihal M, Zhong W, Ahmad N (2009) Role of histone deacetylase SIRT1 in prostate cancer. A target for prostate cancer management via its inhibition? J Biol Chem 284(6): 3823-3832.

70. Jung-Hynes B, Schmit TL, Reagan-Shaw SR, Siddiqui IA, Mukhtar H, et al. (2011) Melatonin, a novel Sirt1 inhibitor, imparts antiproliferative effects against prostate cancer in vitro in culture and in vivo in TRAMP model. J Pineal Res 50(2): 140-149.

71. Satoh A, Brace CS, Rensing N, Cliften P, Wozniak DF, et al. (2013) Sirt1 extends life span and delays aging in mice through the regulation of $\mathrm{Nk} 2$ homeobox 1 in the DMH and LH. Cell Metab 18(3): 416-430.

72. Hardeland R (2017) Future demands concerning the epigenetic relevance of melatonin and the circadian system in gerontology. J Geriatr Med Gerontol 3(4): 036.

73. Rodríguez MI, Escames G, López LC, López A, García JA, et al. (2008) Improved mitochondrial function and increased life span after chronic melatonin treatment in senescent prone mice. Exp Gerontol 43(8): 749756. 


\section{Cell \& Cellular Life Sciences Journal}

74. Gutierrez-Cuesta J, Tajes M, JiménezA, Coto-Montes A, Camins A, et al. (2008) Evaluation of potential prosurvival pathways regulated by melatonin in a murine senescence model. J Pineal Res 45(4): 497505.

75. Hardeland R (2014) Melatonin, noncoding RNAs, messenger RNA stability and epigenetics - evidence, hints, gaps and perspectives. Int J Mol Sci 15(10): 18221-18252.

76. Fu L, Pelicano H, Liu J, Huang P, Lee C (2002) The circadian gene Period2 plays an important role in tumor suppression and DNA damage response in vivo. Cell 111(1): 41-50.

77. Yang W, Kang X, Qin N, Li F, Jin X, et al. (2017) Melatonin protects chondrocytes from impairment induced by glucocorticoids via $\mathrm{NAD}^{+}$-dependent SIRT1. Steroids 126: 24-29.

78. Yang Y, Jiang S, Dong Y, Fan C, Zhao L, et al. (2015) Melatonin prevents cell death and mitochondrial dysfunction via a SIRT1-dependent mechanism during ischemic-stroke in mice. J Pineal Res 58(1): 61-70.

79. Zhao L, An R, Yang Y, Yang X, Liu H, et al. (2015) Melatonin alleviates brain injury in mice subjected to cecal ligation and puncture via attenuating inflammation, apoptosis, and oxidative stress: the role of SIRT1 signaling. J Pineal Res 59(2): 230-239.

80. Carloni S, Favrais G, Saliba E, Albertini MC, Chalon S, et al. (2016) Melatonin modulates neonatal brain inflammation through endoplasmic reticulum stress, autophagy, and miR-34a/silent information regulator 1 pathway. J Pineal Res 61(3): 370-380.

81. Cristòfol R, Porquet D, Corpas R, Coto-Montes A, Serret J, et al. (2012) Neurons from senescenceaccelerated SAMP8 mice are protected against frailty by the sirtuin 1 promoting agents melatonin and resveratrol. J Pineal Res 52(3): 271-281.

82. Volt H, García JA, Doerrier C, Díaz-Casado ME, GuerraLibrero A, et al. (2016) Same molecule but different expression: aging and sepsis trigger NLRP3 inflammasome activation, a target of melatonin. J Pineal Res 60(2): 193-205.

83. Emamgholipour S, Hossein-Nezhad A, Sahraian MA, Sahraian MA, Askarisadr F, et al. (2016) Evidence for possible role of melatonin in reducing oxidative stress in multiple sclerosis through its effect on SIRT1 and antioxidant enzymes. Life Sci 145: 34-41.

84. Li S, Zhang L (2015) Circadian control of global transcription. Biomed Res Int 2015: 187809.

85. Peek CB, Affinati AH, Ramsey KM, Kuo HY, Yu W, et al. (2013) Circadian clock $\mathrm{NAD}^{+}$cycle drives mitochondrial oxidative metabolism in mice. Science 342(6158): 1243417.

86. Masri S (2015) Sirtuin-dependent clock control: New advances in metabolism, aging and cancer. Curr Opin Clin Nutr Metab Care 18(6): 521-527.

87. Song C, Peng W, Yin S, Zhao J, Fu B, et al. (2016) Melatonin improves age-induced fertility decline and attenuates ovarian mitochondrial oxidative stress in mice. Sci Rep 6: 35165.

88. Tamura H, Kawamoto M, Sato S, Tamura I, Maekawa $\mathrm{R}$, et al. (2017) Long-term melatonin treatment delays ovarian aging. J Pineal Res 62(2): 12381.

89. Han L, Wang H, Li L, Li X, Ge J, et al. (2017) Melatonin protects against maternal obesity-associated oxidative stress and meiotic defects in oocytes via the SIRT3-SOD2-dependent pathway. J Pineal Res 63(3): 12431.

90. Song C, Zhao J, Fu B, Li D, Mao T, et al. (2017) Melatonin-mediated upregulation of Sirt3 attenuates sodium fluoride-induced hepatotoxicity by activating the MT1-PI3K/AKT-PGC-1 $\alpha$ signaling pathway. Free Radic Biol Med 112: 616-630.

91. Yu L, Gong B, Duan W, Fan C, Zhang J, et al. (2017) Melatonin ameliorates myocardial ischemia/reperfusion injury in type 1 diabetic rats by preserving mitochondrial function: role of AMPKPGC-1 $\alpha$-SIRT3 signaling. Sci Rep 7: 41337.

92. Zhang M, Lin J, Wang S, Cheng Z, Hu J, et al. (2017) Melatonin protects against diabetic cardiomyopathy through Mst1/Sirt3 signaling. J Pineal Res 63(2): 12418.

93. Zhai M, Li B, Duan W, Jing L, Zhang B, et al. (2017) Melatonin ameliorates myocardial ischemia reperfusion injury through SIRT3-dependent regulation of oxidative stress and apoptosis. J Pineal Res 63(2): 12419. 


\section{Cell \& Cellular Life Sciences Journal}

94. Chang HC, Guarente L (2013) SIRT1 mediates central circadian control in the SCN by a mechanism that decays with aging. Cell 153(7): 1448-1460.

95. Carthew RW, Sontheimer EJ (2009) Origins and mechanisms of miRNAs and siRNAs. Cell 136(4): 642655.

96. Bartel DP (2009) MicroRNAs: target recognition and regulatory functions. Cell 136(2): 215-233.

97. Krol J, Loedige I, Filipowicz W (2010) The widespread regulation of microRNA biogenesis, function and decay. Nat Rev Genet 11(9): 597-610.

98. Da Costa Martins PA, De Windt LJ (2012) Targeting microRNA targets. Circ Res 111(5): 506-508.

99. Berezikov E, Chung WJ, Willis J, Cuppen E, Lai EC (2007) Mammalian mirtron genes. Mol Cell 28(2): 328-336.

100. Zhang X, Cozen AE, Liu Y, Chen Q, Lowe TM (2016) Small RNA modifications: integral to function and disease. Trends Mol Med 22(12): 1025-1034.

101. Nishikura K (2016) A-to-I editing of coding and noncoding RNAs by ADARs. Nat Rev Mol Cell Biol 17(2): 83-96.

102. Liu HY, Huang CM, Hung YF, Hsueh YP (2015) The microRNAs Let7c and miR21 are recognized by neuronal Toll-like receptor 7 to restrict dendritic growth of neurons. Exp Neurol 269: 202-212.

103. Paschon V, Takada SH, Ikebara JM, Sousa E, Raeisossadati R, et al. (2016) Interplay between exosomes, microRNAs and toll-like receptors in brain disorders. Mol Neurobiol 53(3): 2016-2028.

104. Chen JJ, Zhao B, Zhao J, Li S (2017) Potential roles of exosomal microRNAs as diagnostic biomarkers and therapeutic application in Alzheimer's disease. Neural Plast 2017: 7027380.

105. Meldolesi J (2016) Ectosomes and exosomes-Two extracellular vesicles that differ only in some details. Biochem Mol Biol J 2(1): 03.

106. Kim KM, Abdelmohsen K, Mustapic M, Kapogiannis D, Gorospe M (2017) RNA in extracellular vesicles. Wiley Interdiscip Rev RNA 8(4): e1413.
107. Hansen TB, Jensen TI, Clausen BH, Bramsen JB, Finsen B, et al. (2013) Natural RNA circles function as efficient microRNA sponges. Nature 495(7441): 384-388.

108. Ahadi A, Brennan S, Kennedy PJ, Hutvagner G, Tran N (2016) Long non-coding RNAs harboring miRNA seed regions are enriched in prostate cancer exosomes. Sci Rep 6: 24922.

109. Hardeland R (2018) Interactions of melatonin and microRNAs. Biochem Mol Biol J 4(1): 07.

110. Wang $\mathrm{X}$, Wang $\mathrm{ZH}, \mathrm{Wu} \mathrm{YY}$, Tang $\mathrm{H}$, Tan $\mathrm{L}$, et al. (2013) Melatonin attenuates scopolamine-induced memory/synaptic disorder by rescuing EPACs/miR124/Egr1 pathway. Mol Neurobiol 47(1): 373-381.

111. Zhao Y, Zhao R, Wu J, Wang Q, Pang K, et al. (2018) Melatonin protects against $A \beta$-induced neurotoxicity in primary neurons via miR132/PTEN/AKT/FOXO3a pathway. Biofactors. [Epub ahead of print, Jan 11]; doi: 10.1002/biof.1411.

112. Meng F, Dai E, Yu X, Zhang Y, Chen X, et al. (2013) Constructing and characterizing a bioactive small molecule and microRNA association network for Alzheimer's disease. J R Soc Interface 11(92): 20131057.

113. Fu S, Zhao H, Zheng Z, Li J, Zhang W (2014) Melatonin regulating the expression of miRNAs involved in hair follicle cycle of Cashmere goat's skin. Yi Chuan 36(12): 1235-1242.

114. Cai B, Ma W, Bi C, Yang F, Zhang L, et al. (2016) Long noncoding RNA H19 mediates melatonin inhibition of premature senescence of c-kit cardiac progenitor cells by promoting miR-675. J Pineal Res 61(1): 8295.

115. Wu Z, Qiu X, Gao B, Lian C, Peng Y, et al. (2018) Melatonin-mediated miR-526b-3p and miR-590-5p upregulation promotes chondrogenic differentiation of human mesenchymal stem cells. J Pineal Res e12483.

116. Sohn EJ, Won G, Lee J, Lee S, Kim SH (2015) Upregulation of miRNA3195 and miRNA374b mediates the anti-angiogenic properties of melatonin in hypoxic PC-3 prostate cancer cells. J Cancer 6(1): 19-28. 


\section{Cell \& Cellular Life Sciences Journal}

117. Lee SE, Kim SJ, Youn J-P, Hwang SY, Park CS, et al. (2011) MicroRNA and gene expression analysis of melatonin-exposed breast cancer cell lines indicating involvement of the anticancer effect. J Pineal Res 51(3): 345-352.

118. Gu J, Lu Z, Ji C, Chen Y, Liu Y, et al. (2017) Melatonin inhibits proliferation and invasion via repression of miRNA-155 in glioma cells. Biomed Pharmacother 93: 969-975.

119. Cheng L, Liu J, Liu Q, Liu Y, Fan L, et al. (2017) Exosomes from melatonin treated hepatocellularcarcinoma cells alter the immunosupression status through STAT3 pathway in macrophages. Int J Biol Sci 13(6): 723-734.

120. Flemström G, Sjöblom M (2005) Epithelial cells and their neighbors. II. New perspectives on efferent signaling between brain, neuroendocrine cells, and gut epithelial cells. Am J Physiol Gastrointest Liver Physiol 289(3): G377-G380.

121. Bengtsson MW, Jedstedt G, Flemström G (2007) Duodenal bicarbonate secretion in rats: stimulation by intra-arterial and luminal guanylin and uroguanylin. Acta Physiol (Oxf) 191(4): 309-317.

122. Hardeland R (2017) A short overview of duodenal signaling by melatonin. In: Mini-Reviews in Recent Melatonin Research (Hardeland R, Ed), Cuvillier, Göttingen, Germany pp: 22-25.

123. Hardeland R (2017) Intercellular communication via exosomal and ectosomal microRNAs: Facing a jungle of countless microRNAs and targets. In: MiniReviews in Recent Melatonin Research (Hardeland R, Ed), Cuvillier, Göttingen, Germany pp: 109-122. 